Hybrid Live Coding Interfaces: performance and craft • Hybrid Live Coding Interfaces: performance and craft

\title{
Agency in Live Coded Choreography
}

Kate Sicchio

Published on: Jul 07, 2020

DOI: 10.21428/40e92d8f.bcdee0a5

License: Creative Commons Attribution 4.0 International License (CC-BY 4.0). 
There has been algorithmic choreography as long as there have been computers, with the first article published on the subject in 1966 in Dance Magazine. Different approaches from animating stick figures, to abstract instructions have been used to create dances with computers. The end results often do not stop at this computation, but rather an additional interpretation by the dancers. Even the strictest following of rules or choreography still will create different approaches, movement intentions, or relationships of the body in space and time. This presentation will discuss the human interpreter, the role of agency and how live coded choreography will always be removed from its code in its final presentation. 